\title{
Weit weg und doch nah - zwei Technikkonzepte im Familieneinsatz
}

\author{
Wasili Adamow ${ }^{1}$, Patrick Beedgen ${ }^{1}$, Eva Lenz ${ }^{2}$, Thies Schneider ${ }^{2}$, Kirstin \\ Kohler $^{1}$, Marc Hassenzahl ${ }^{2}$ \\ Fakultät für Informatik I User Experience \& Interaction Design, Hochschule Mannheim ${ }^{1}$ \\ Fachbereich Gestaltung I Erlebnis und Interaktion, Folkwang Universität der Künste ${ }^{2}$
}

\section{Zusammenfassung}

In enger Zusammenarbeit mit Familien, die von einander räumlich getrennt leben, haben wir uns damit beschäftigt, wie Technik gestaltet sein muss, um das Gefühl der Verbundenheit über die Distanz zu stärken. Stellvertretend für eine Reihe verschiedener Konzepte, die wir für diesen Kontext gestaltet, entwickelt und in den Familien erprobt haben, beschreiben wir hier zwei Konzepte genauer. Wir stellen die wichtigsten Gestaltungsentscheidungen dar und deren Motivation basierend auf den individuellen Bedürfnissen der Familien.

\section{Nähe auf Distanz}

Im familiären Kontext erlebte Nähe spielt für das Wohlbefinden von Menschen eine wichtige Rolle. Doch nicht selten wird dieses Erleben durch räumliche Distanz erschwert. Einer interventions- und gestaltungsorientierten Forschungsmethodik folgend, haben wir verschiedene Konzepte erarbeitet, die explizit auf die individuellen Erfordernisse von fünf verschiedenen Familien eingehen. Dabei haben alle Konzepte das gemeinsame Gestaltungsziel, Verbundenheitsgefühle auch über die Distanz zu fördern. Es ging nicht darum, neue Technologien zu entwickeln, sondern vielmehr, bestehende Technik leichtgewichtig neu zu kombinieren und zu konfigurieren. Neue Erlebnisse sind das Ziel, erarbeitet aus vorhandenen Technologien. In diesem Beitrag stellen wir beispielhaft für diese Arbeit zwei Konzepte und ihre Gestaltungsentscheidungen vor. Beide Konzepte sind in Form von funktionalen Prototypen umgesetzt und waren für mindestens 10 Tage bei ,,ihren“ Familien im Einsatz. 


\section{Der „Ich zeig dir was“ - Bilderrahmen}

Ein wiederkehrendes Thema in der Kommunikation zwischen Generationen ist die Suche nach einem gemeinsamen Gesprächsthema. Alltagstrivialitäten scheinen zu nichtig, und gemeinsame Anknüpfungspunkte gibt es wenige. So berichtete Kathrin von den Telefonaten mit ihrer unter Demenz leidenden Mutter Agnes, dass diese sich oft auf reine "Kontrollgespräche" reduzierten, die kaum mehr als die Einnahme der Tabletten oder andere Hinweise thematisierten.

Mit Hilfe eines digitalen Bilderrahmens, der bei Mutter und Tochter neben dem Telefon steht, wollen wir die Gespräche zwischen Kathrin und Agnes mit Bildern anreichern, um der Tochter Inhalte für Erzählungen zu liefern und die Mutter auf diese Weise am Alltag ihrer Tochter teilhaben zu lassen (siehe Abbildung 1). Als leidenschaftliche Photographin macht Kathrin viele Bilder im Alltag oder im Urlaub, die sie in digitaler Form auf ihrem Computer verwaltet. Unser Konzept bietet einen Fotoupload an, mit dem sie ausgewählte Bilder zur Anzeige auf dem Bilderrahmen auswählen kann. Die Bilder kann sie dabei mit einem kurzen Text anreichern. Sie werden anschließend auf beide Bilderrahmen, sowohl bei der Tochter als auch bei der Mutter, hochgeladen. Wenn Kathrin nun Agnes anruft und erzählt, dass sie vor kurzem aus dem Urlaub zurückgekommen ist, hat sie genug Gesprächsstoff. Kathrin und ihre Mutter sehen das gleich Bild von Kathrins Urlaub im Bilderrahmen. Um zum nächsten Bild zu wechseln, wischt Kathrin über das Display ihres Rahmens. Auf diese Weise steuert sie synchron auch die Anzeige ihrer Mutter. Sie kann so ihre Mutter während des Telefonats durch ihr Album führen.


Abbildung 1: Der „Ich zeig dir was“ - Bilderrahmen bei der Benutzung während eines Telefonats

Wichtig an diesem Konzept ist die asymmetrische Bedienung durch Swipen: Zwei verbundene Bilderrahmen funktionieren nach dem Master-Slave Prinzip. Ein Bilderrahmen hat die Kontrolle über die angezeigten Bilder, während der andere Bilderrahmen eine rein passive Rolle einnimmt. Wenn der „Master“-Bilderrahmen die Bilder wechselt, wird auf dem „Slave"-Bilderrahmen das gleiche Bild angezeigt. Der Wechsel erfolgt in unter einer Sekunde, um die Verwendung während eines Telefonats zu ermöglichen. Andere digitale Bilderrahmen, wie bspw. das von Kim \& Zimmerman (2006) vorgestellte System „Cherish“ oder das 
von Daalsgard et al. (2007) entwickelte System „eKISS“ verfügen zwar über die Textannotationen, stellen aber keine Möglichkeit zur Verfügung, die Anzeige über die Distanz zu steuern.

Technisch haben wir den Bilderrahmen mit zwei Android-Tabletts realisiert, deren Zugänge durch den Holzbilderrahmen verborgen sind, sodass die Tabletts einzig für den hier beschriebenen Zweck genutzt werden konnten. Beim „Slave“-Rahmen ist die TouchFunktionalität des Tabletts zusätzlich durch eine Plexiglasscheibe abgesperrt. Die Tabletts kommunizieren über eine internetbasierte Verbindung vermittelt durch einen Server. Die aktuellen Bilder werden aus Performanzgründen lokal vorgehalten.

\section{Die Bilderbox}

Immer häufiger wird heutzutage das Problem der Vereinbarkeit von Beruf und Familie thematisiert und es kommt vor, dass neben der Arbeit zu wenig Zeit für die Familie bleibt. Auch bei Familie Peters gibt es eine solche Situation: Der Vater arbeitet tagsüber in einer Bank, während seine Söhne im Kindergarten beziehungsweise zuhause sind. Sowohl Vater als auch Kinder wünschen sich eine Möglichkeit kurz und unkompliziert miteinander zu kommunizieren. Allerdings können die Kinder die Kommunikation nicht selbstständig initiieren, und der Vater hat oft das Gefühl, nicht genug Zeit für ein Gespräch zu haben

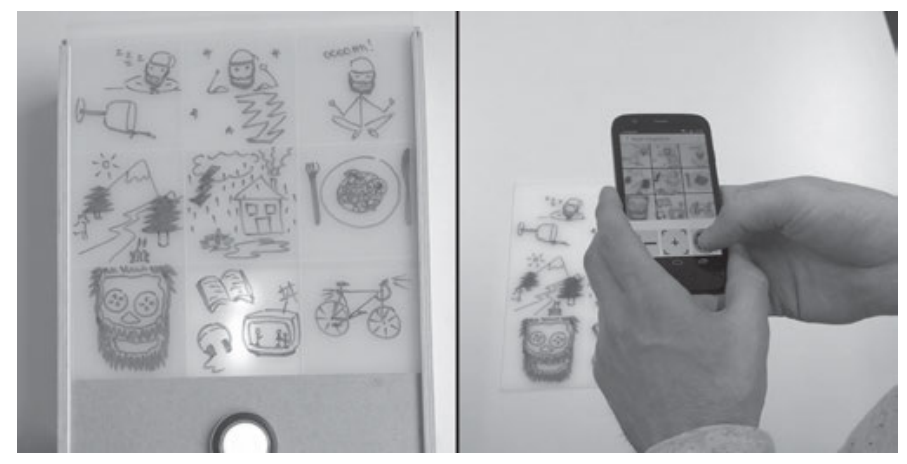

Abbildung 2: Die Bilderbox im Einsatz mit dem dazugehörigen Smartphone

Die Bilderbox (siehe Abbildung 2) befähigt die Kinder eigenständig mit dem Vater in Kontakt zu treten und hält gleichzeitig den Aufwand für den Vater gering, persönliche Nachrichten an die Kinder zu senden. Die Box verbleibt zuhause bei den Kindern. Sie verfügt über neun beleuchtete Felder und einen Knopf. Die Felder selbst sind mit einer Milchglasscheibe verdeckt, auf die mit einem Stift gemalt werden kann. So können die Kinder zusammen mit dem Vater verschiedene Symbole vereinbaren und aufmalen, welche als Grundlage für die Nachrichten dienen. Die hierbei entstehende Offenheit soll die Aneignung fördern (Gaver et al. 2003). 
Die Kinder können tagsüber den „Status“ des Vaters anfordern, indem sie den Knopf auf der Box drücken. Die Box signalisiert durch Blinken eine Warteanimation, während der Vater eine Benachrichtigung auf sein Smartphone erhält. Die Benachrichtigung führt ihn in die App, in der er in einer ihm freigestellten Reihenfolge die Symbole antippen kann. (Diese wurden vorher fotografiert.) So entsteht eine Nachricht, die er direkt an die Kinder schickt. Die Box zeigt infolgedessen die Symbole hell leuchtend, in der gedrückten Reihenfolge. Diese Nachricht bleibt auf der Box für eine definierte Zeit aktiv, bis die Kinder eine neue Nachricht anfordern (oder der Vater eine neue Nachricht schickt).

Wie bei der „Lovers“ Box“ (Thieme et al. 2011) zielt auch dieses Konzept darauf ab, zwei Seiten der Kommunikation durch Technik mit eigenen, persönlichen Inhalten zu verbinden. So entstehen zusätzliche Interaktionspunkte zwischen Vater und den Kindern, wie das gemeinsame Erstellen und Aushandeln von Symbolen und Nachrichten. Gleichzeitig entlasten einige Restriktionen den Vater in seinem Arbeitsumfeld: So können Anfragen nur alle zwei Stunden gestellt werden, wodurch ein überlegtes Anfordern nötig ist.

Für die Umsetzung wurde ein Einplatinencomputer in der Box verbaut, der verschiedene LEDs und den Knopf über eine eigene Schaltung ansteuert. Beim Vater kam ein AndroidSmartphone mit einer eigens dafür erstellten App zum Einsatz. Die Kommunikation zwischen Box und App erfolgte synchron über einen Server.

Beide Konzepte haben zum Ziel, neue Formen technikvermittelter Verbundenheit zu etablieren und zu erproben. Im Vordergrund steht die einfühlsame Gestaltung der Konzepte, ihre Entwicklung aus dem spezifischen Nutzungskontext heraus, und die Frage, ob die erhofften Erlebnisse sich auch realisieren. Die Umsetzung folgt den Prinzipien von Offenheit und Einfachheit. Im Projekt setzen wir auf frei verfügbare Technologien und auf Konzepte, die zwar reichhaltig im Erleben, aber möglichst einfach in der Umsetzung sind. Konzeptvideos zu beiden Konzepten finden sich unter https://vimeo.com/129546708.

\section{Danksagung}

Finanzierung durch das BMBF: Projekt „Nähe auf Distanz“ (Förderkz.: 16SV7093K)

\section{Literaturverzeichnis}

Dalsgaard, T,, Skov, M.B. \& Thomassen, B.R. (2007). eKISS: sharing experiences in families through a picture blog. In Proceedings of the 21st British HCI Group Annual Conference on People and Computers: (BCS-HCI '07). Vol. 1. British Computer Society, Swinton, UK, 67-75.

Gaver, W.W., Beaver, J. \& Benford, B. (2003). Ambiguity as a resource for design. In Proceedings of the SIGCHI Conference on Human Factors in Computing Systems (CHI '03). ACM, New York, NY, USA, 233-240.

Kim, J. \& Zimmerman, J (2006). Cherish: smart digital photo frames for sharing social narratives at home. In CHI '06 Extended Abstracts on Human Factors in Computing Systems (CHI EA '06). ACM, USA, 953-958.

Thieme, A., Wallace, J., Thomas, J., Chen, K.L., Krämer, N. \& Olivier, P. (2011). Lovers' box: Designing for reflection within romantic relationships. Int. J. Hum.-Comput. Stud. 69, 5 , 283-297. 